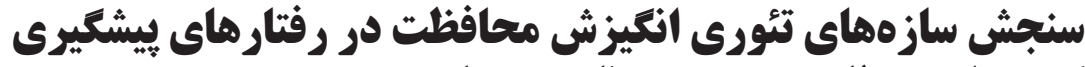

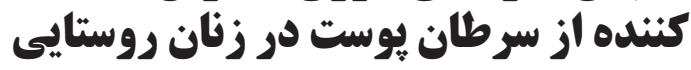

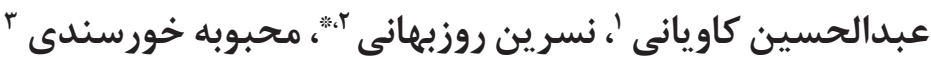

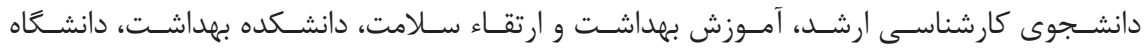

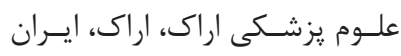

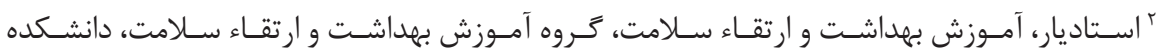

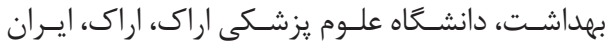

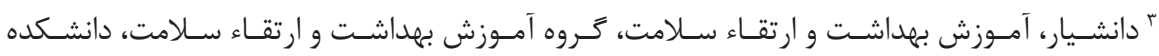

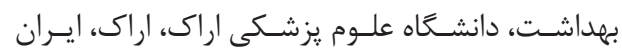

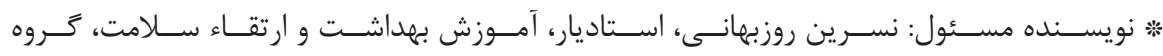

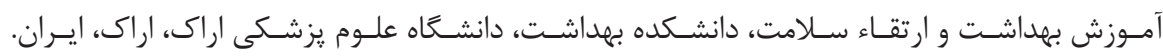

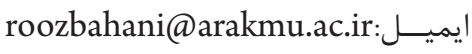

DOI: $10.21859 / \mathrm{nmj}-24043$

קكيده

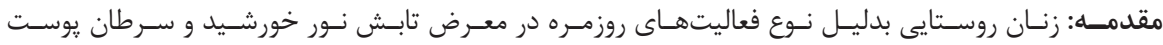

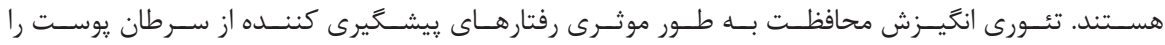

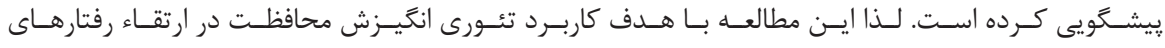

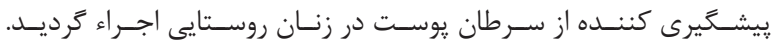

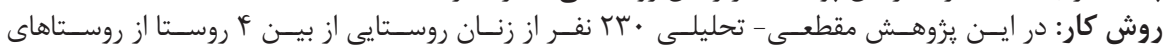

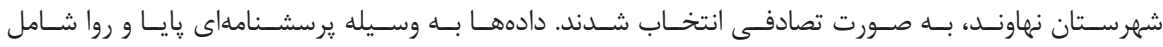

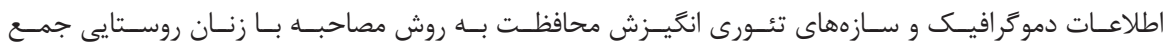

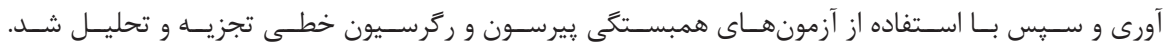

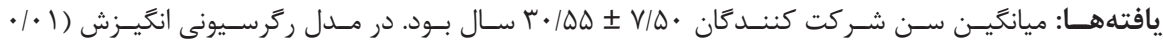

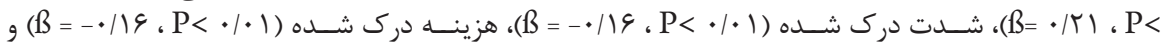

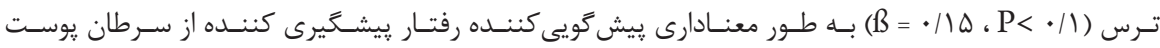

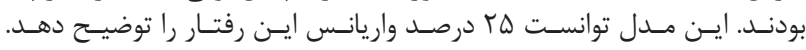

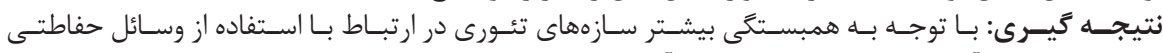

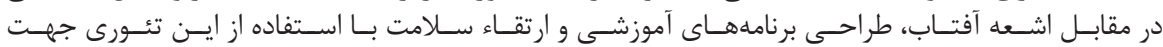

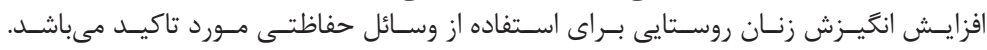

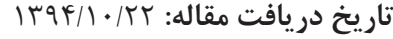
تاريخ يذيرش مقاله:

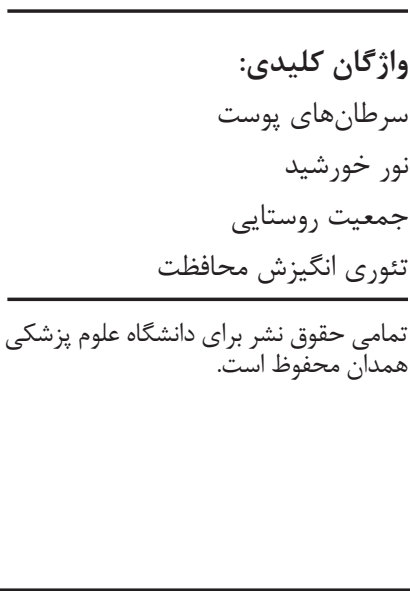

ميـزان هشــار دهنــده اسـت و بــر اسـاس گـزارش ســازمان جهانـى

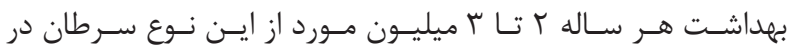

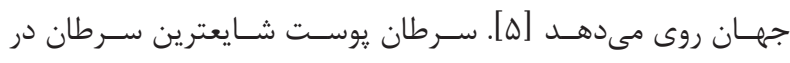

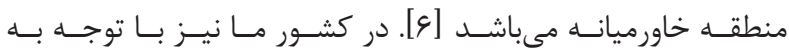

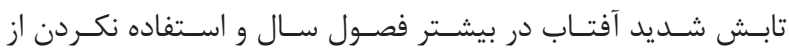

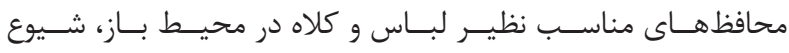

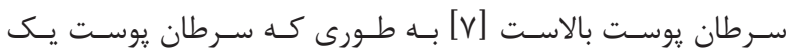
معضـل بهداشـت عمومسى در كشـور مىباشـــ [N]. مطالعاتـى نيـز

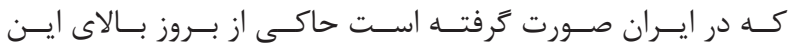

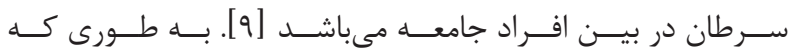

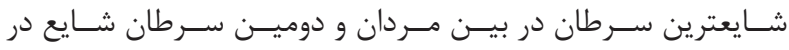

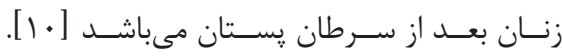

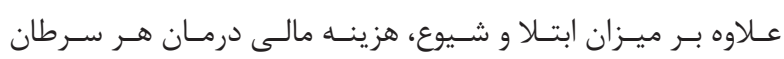

سـرطان يوسـت شـايعترين سـرطان انسـان در جهــان اسـت كــهـ

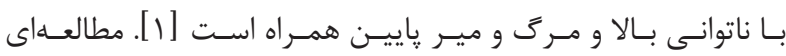

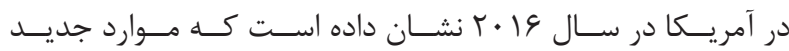

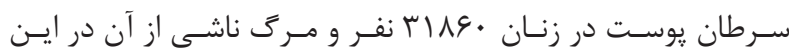

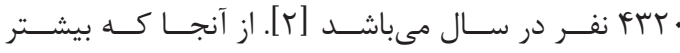

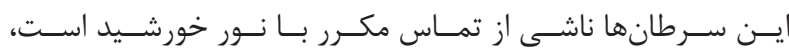

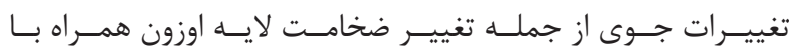

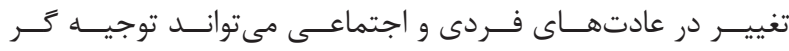

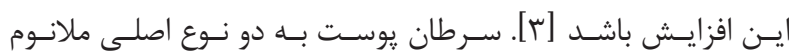

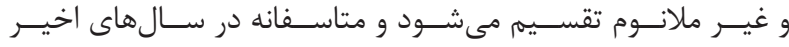

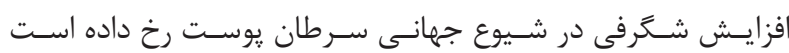

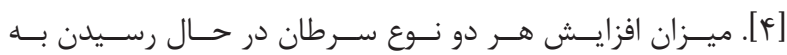




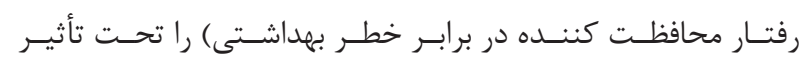

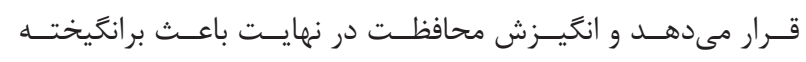

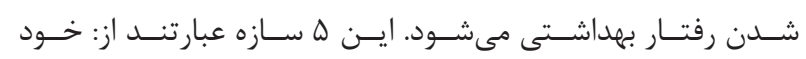

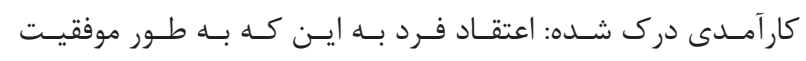

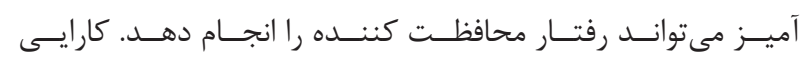

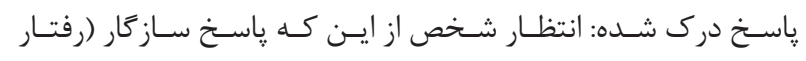

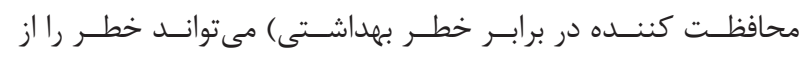

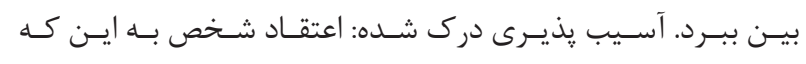

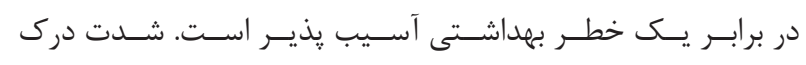

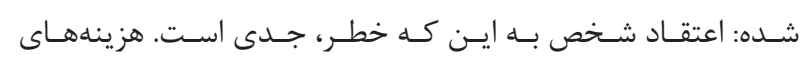

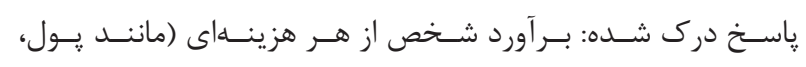

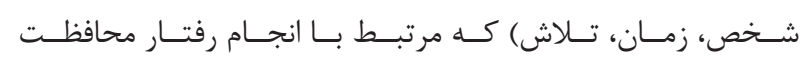

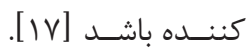

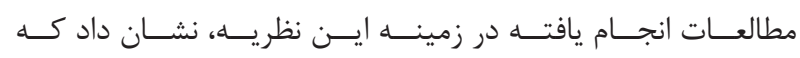

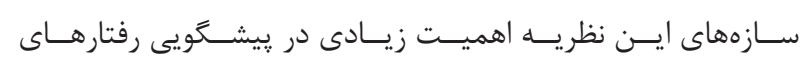

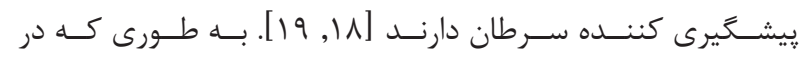

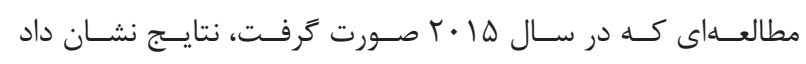

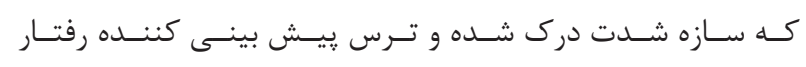

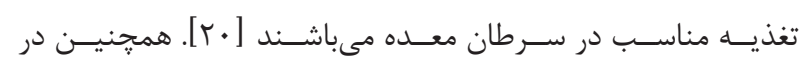

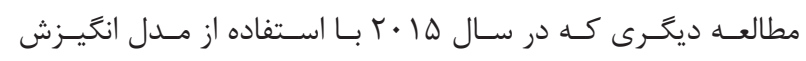

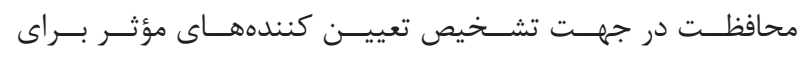

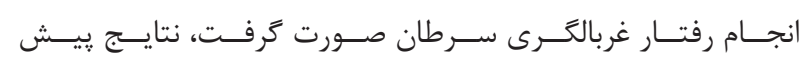

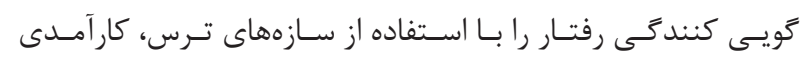

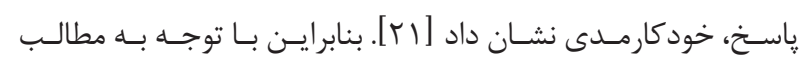

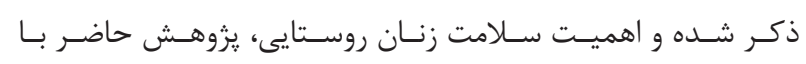

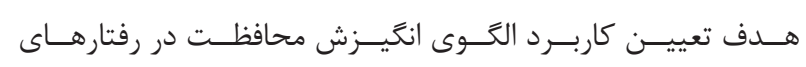

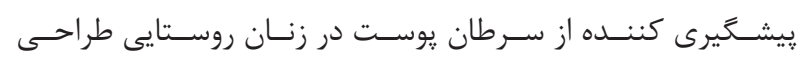
و اجــراء ترديـد.

\section{روش كار}

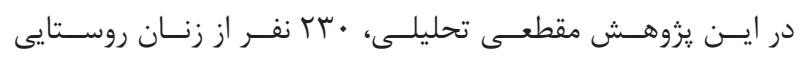

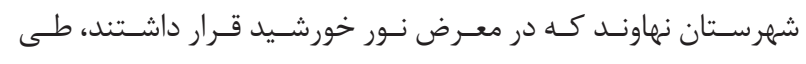

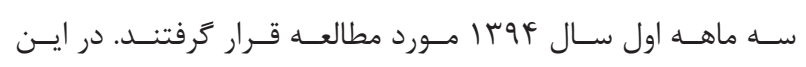

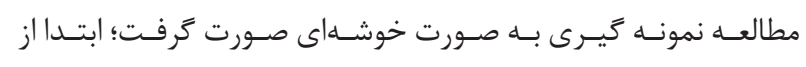

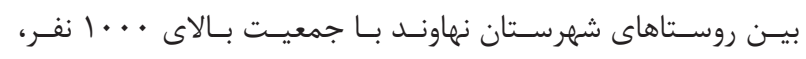

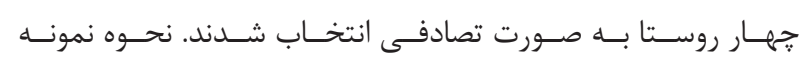

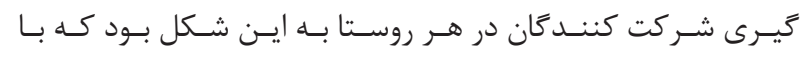

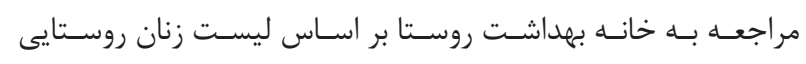

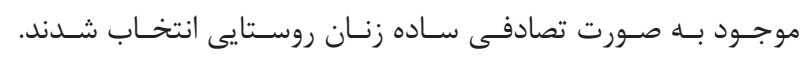

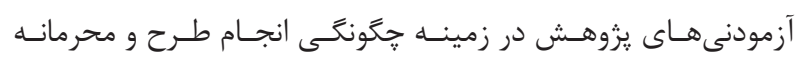

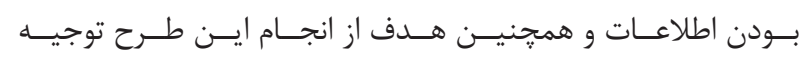

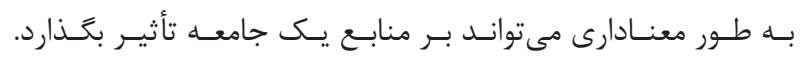

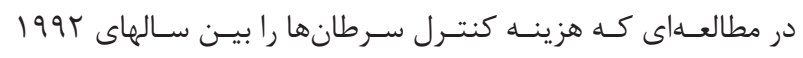

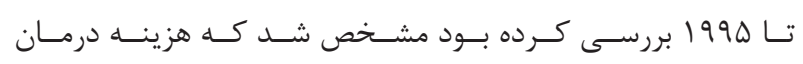

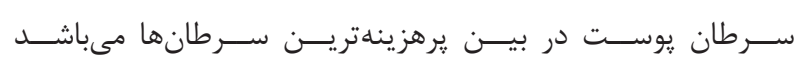

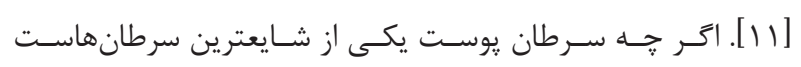

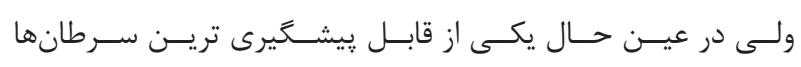

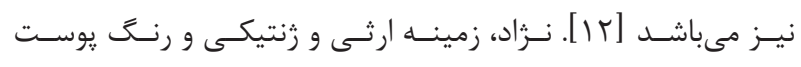

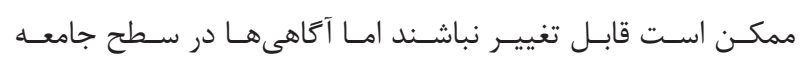

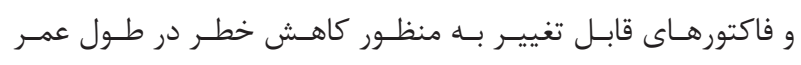

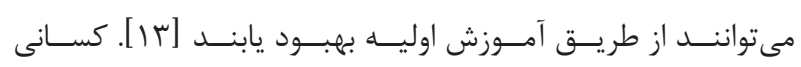

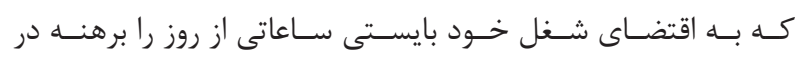

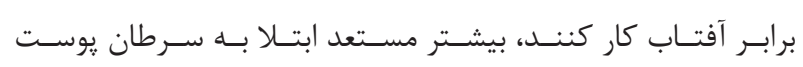

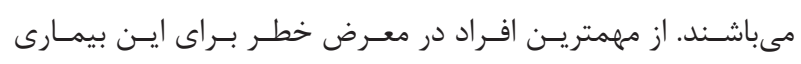

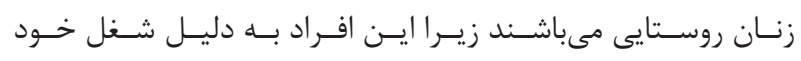

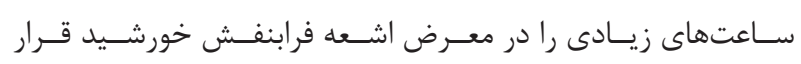

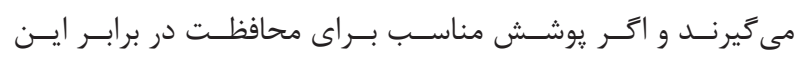

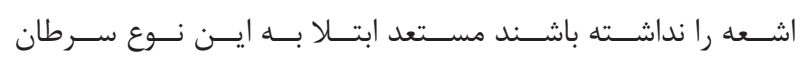

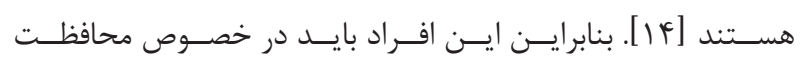

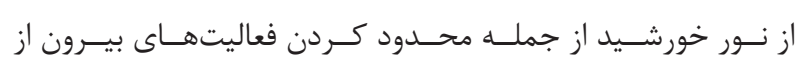

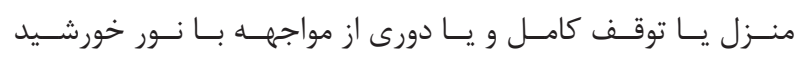

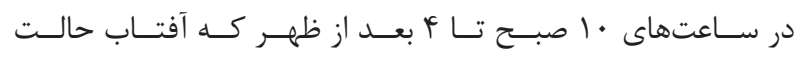

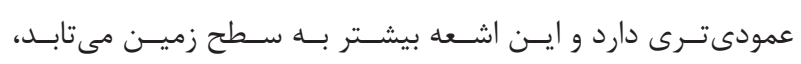

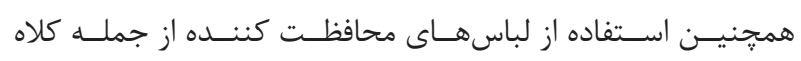

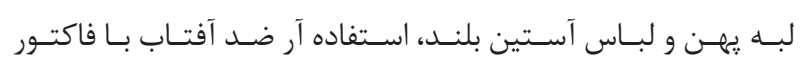

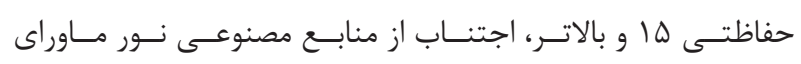

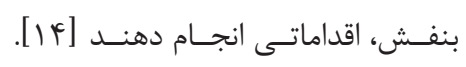

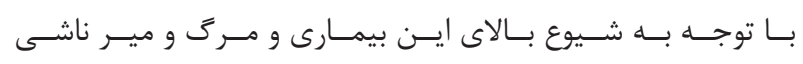

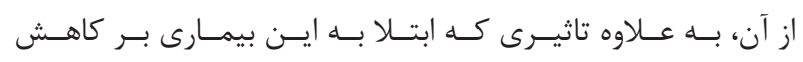

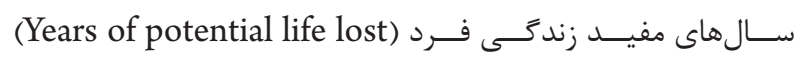

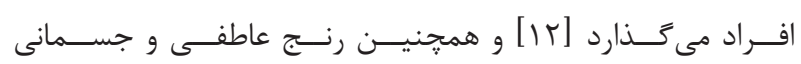

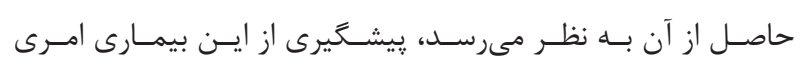

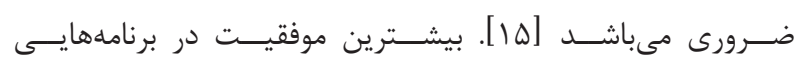

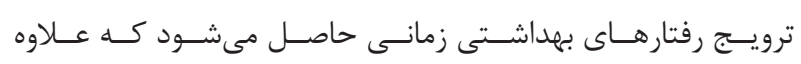

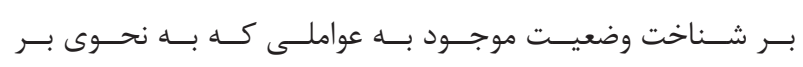

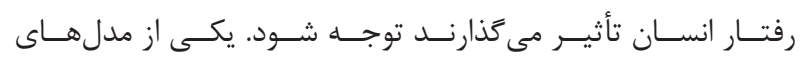

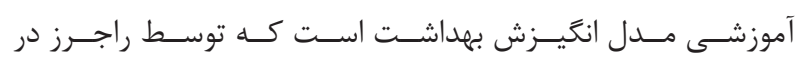

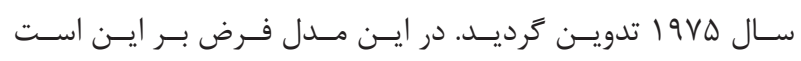

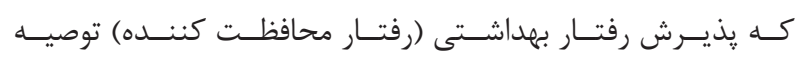

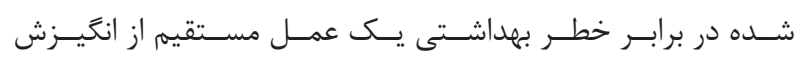

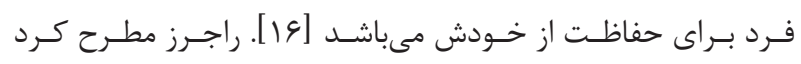

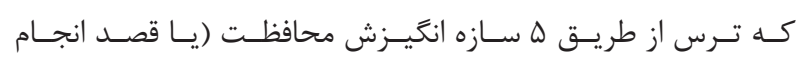


مــورد ارزيابسى قـرار دادنــد و در نهايـت نتايسج حاصلـه محاســبه و

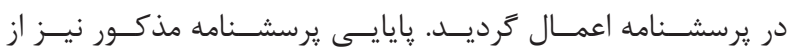

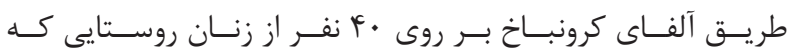

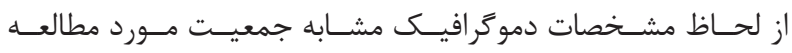

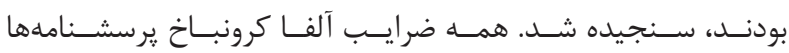

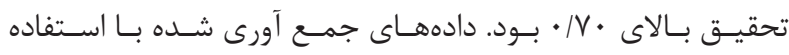

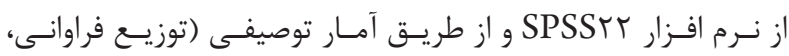

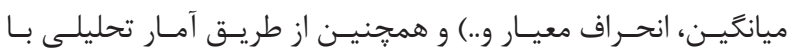

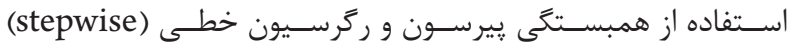

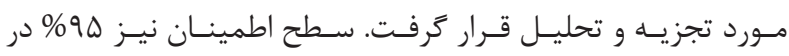

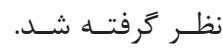

\begin{tabular}{|c|c|}
\hline \multicolumn{2}{|c|}{ جدول ا: اطلاعات دموكرافيك زنان روستايى مورد مطالعه } \\
\hline تعداد (درصد) & متغير \\
\hline & 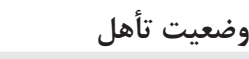 \\
\hline$r f(1 \cdot / f)$ & مجرد \\
\hline$r \cdot r(\Lambda V / \Lambda)$ & متأهل \\
\hline \multirow[t]{2}{*}{$f(1 / V)$} & بيوه \\
\hline & وضعيت تحصيلات \\
\hline $19(V)$ & 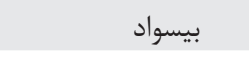 \\
\hline$q \wedge(F / / 9)$ & ابتدايى \\
\hline $\operatorname{Fr}(\mid \Lambda / r)$ & راهنمايى \\
\hline $\operatorname{Fe}(19 / 1)$ & دبيرستان \\
\hline $\operatorname{rr}(1 \cdot)$ & دييلم \\
\hline \multirow[t]{2}{*}{$V(r)$} & دانشَاه \\
\hline & 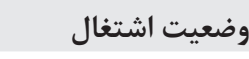 \\
\hline$r \cdots(\Lambda \vee)$ & 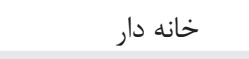 \\
\hline$f(1 / V)$ & 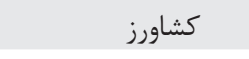 \\
\hline$r(1 / \mu)$ & 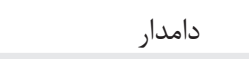 \\
\hline \multirow[t]{2}{*}{$r r(1 \cdot)$} & 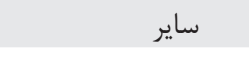 \\
\hline & 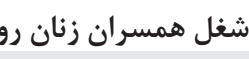 \\
\hline$t \wedge(\mid r / t)$ & 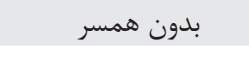 \\
\hline$\Delta \cdot(Y) / V)$ & 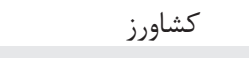 \\
\hline$F(1 / V)$ & 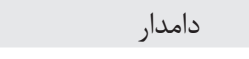 \\
\hline $\mid V(V / F)$ & 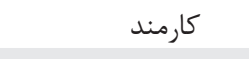 \\
\hline $99(Y N / V)$ & 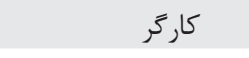 \\
\hline \multirow{2}{*}{$\varepsilon \Delta(Y \Lambda / r)$} & 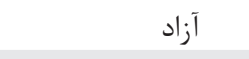 \\
\hline & سابقه آفتاب سوختخح \\
\hline $\mid \Delta F(G V)$ & بله \\
\hline Vब(Tr) & خير \\
\hline
\end{tabular}

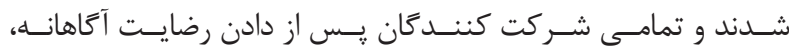

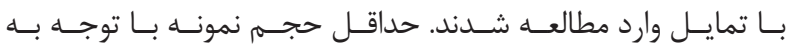

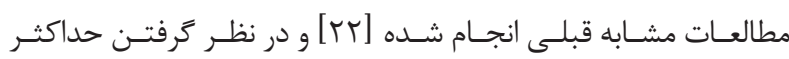

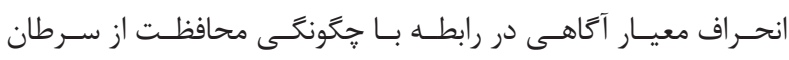

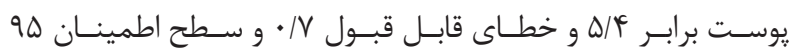

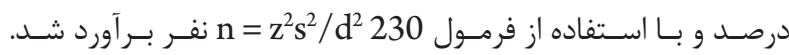

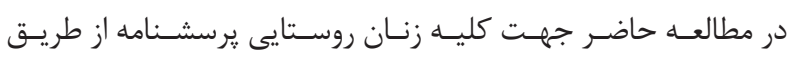
مصاحبـه تكميـل كرديسد.

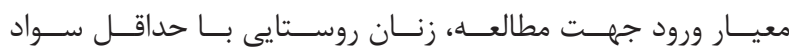

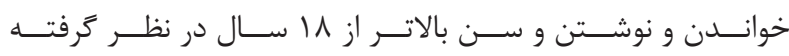

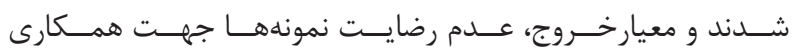

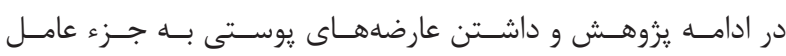

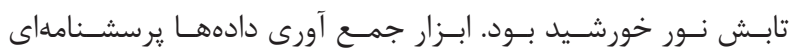

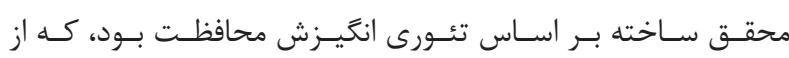

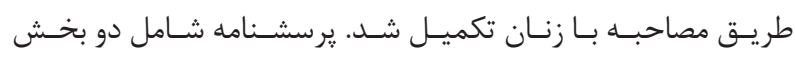

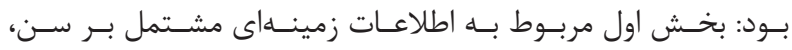

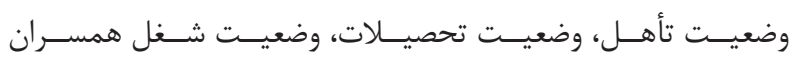

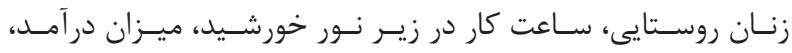

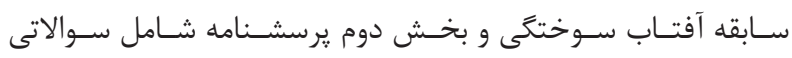

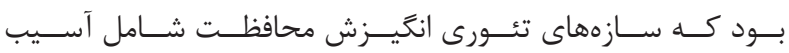

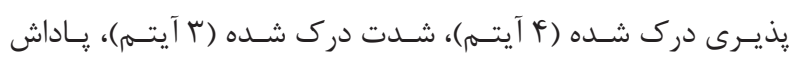

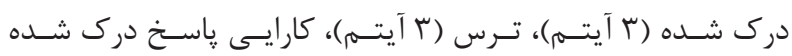

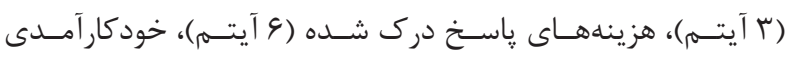

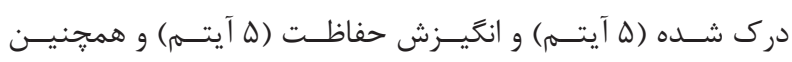

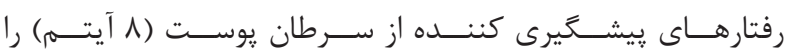

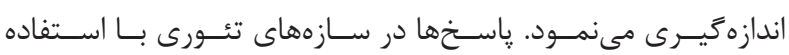

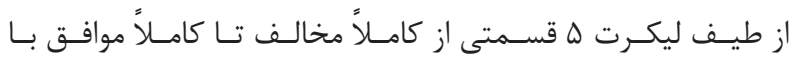

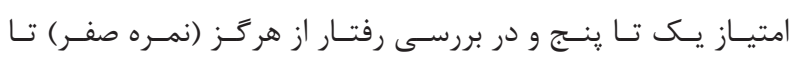

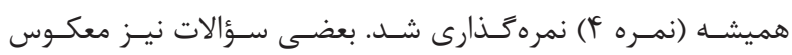

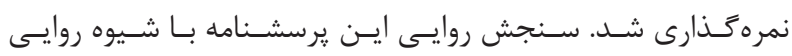

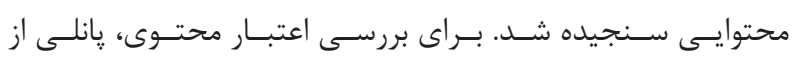

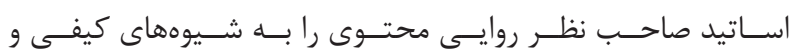

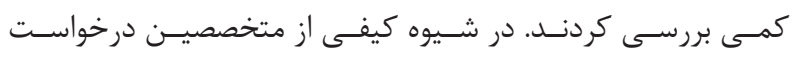

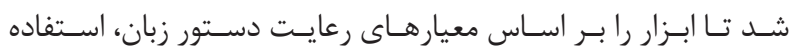

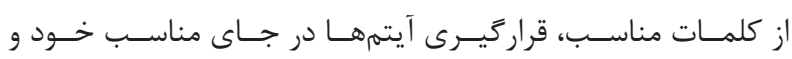

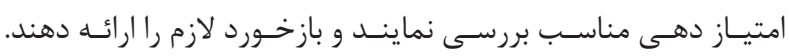

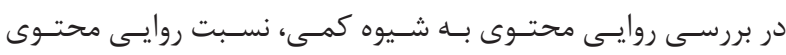

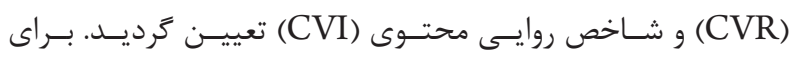

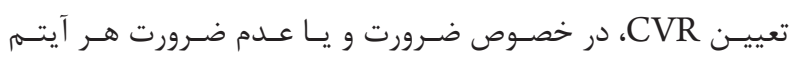

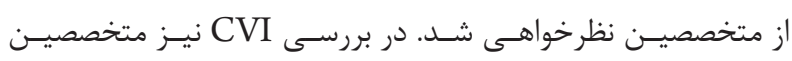

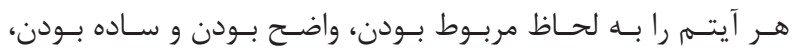




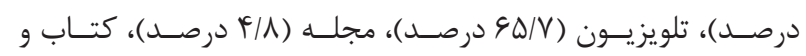

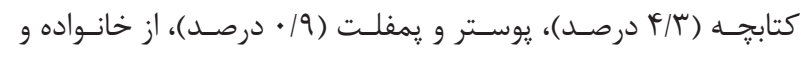

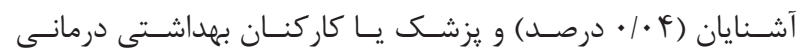

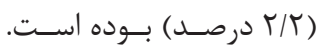

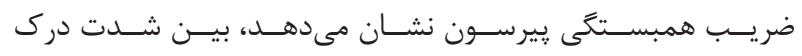

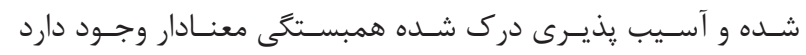

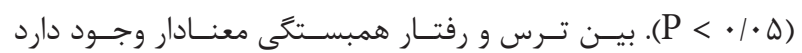

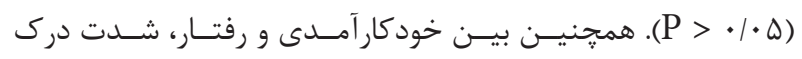

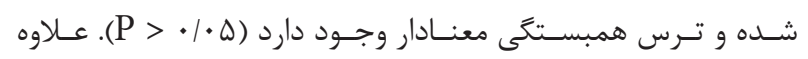

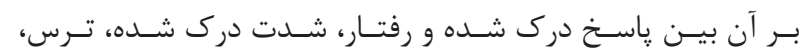

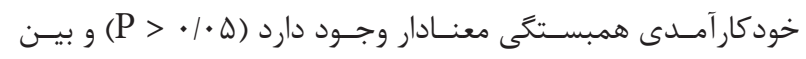

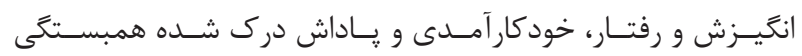

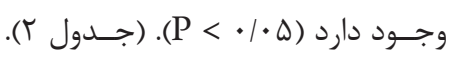

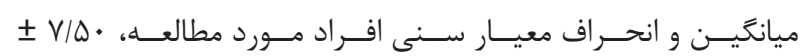

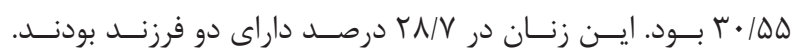

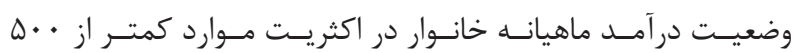

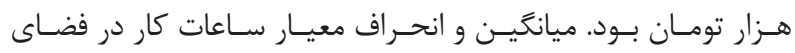

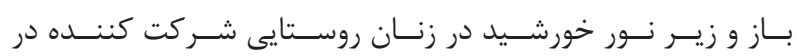

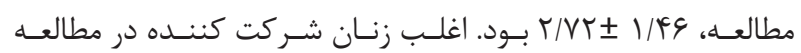

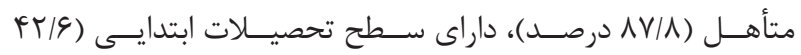

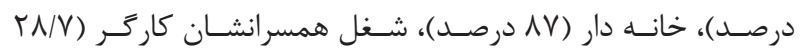

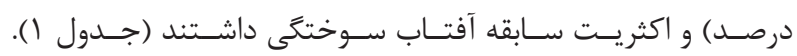

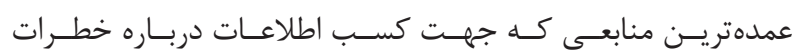

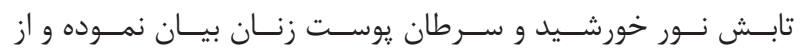

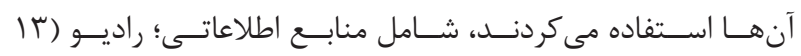

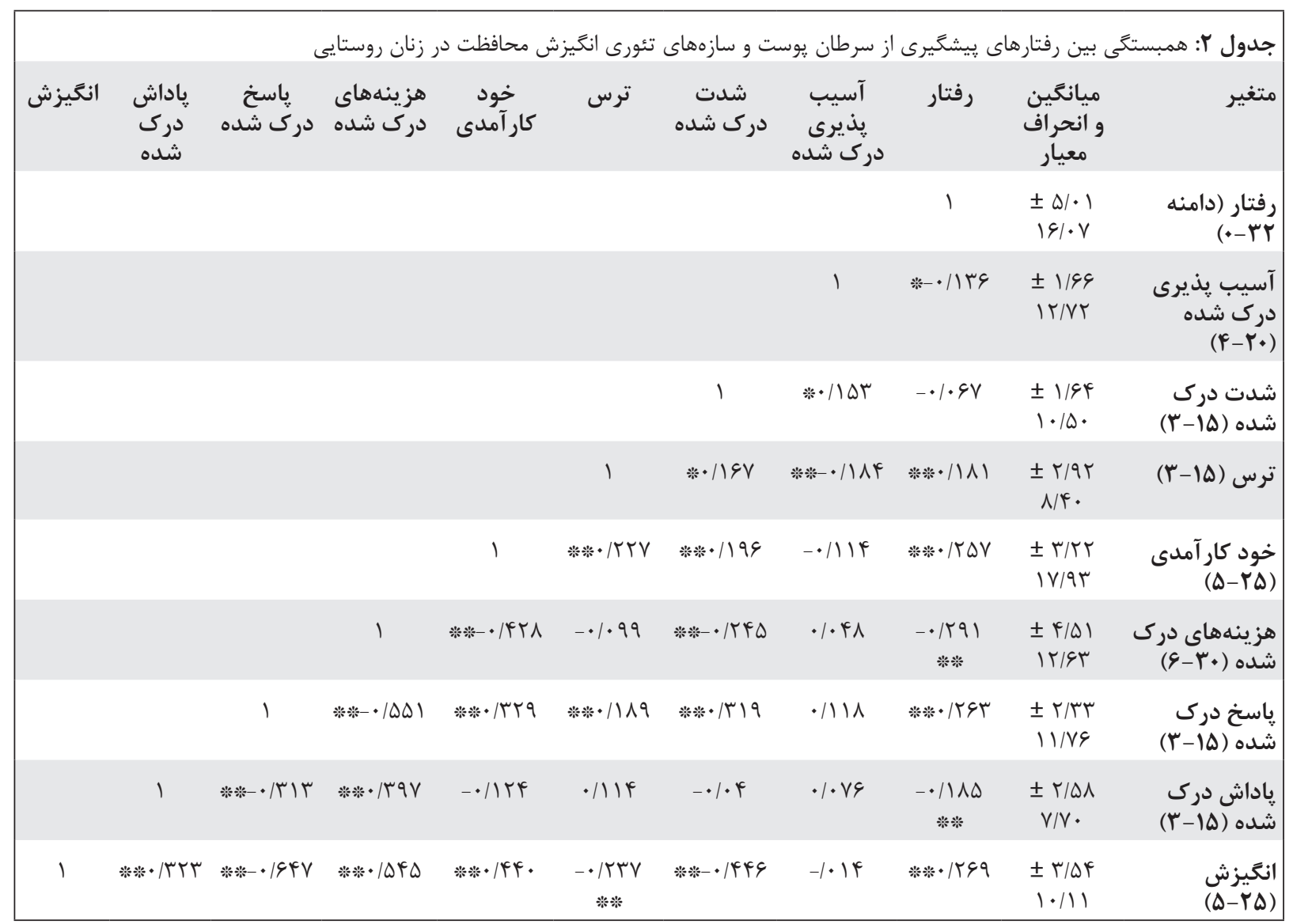

$\mathrm{P}<\cdot 1 \cdot \Delta *$ $\mathrm{P}<\cdot / \cdot 1$ ***

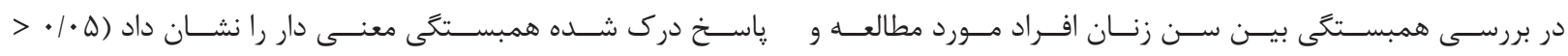

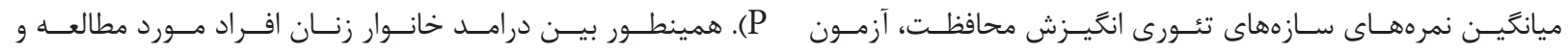

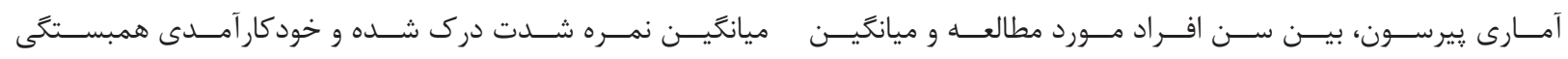

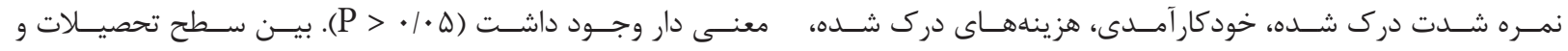




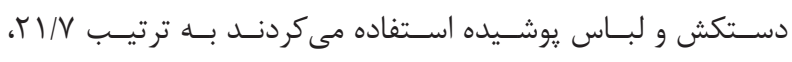
از

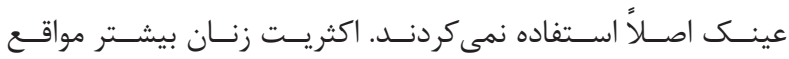

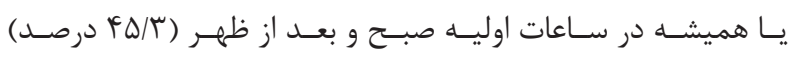

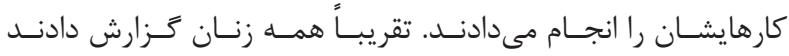

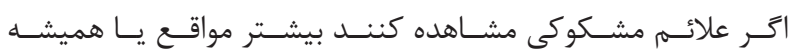

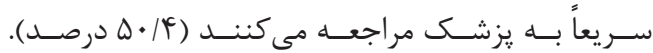

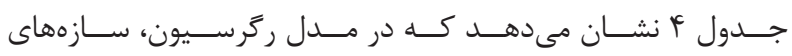

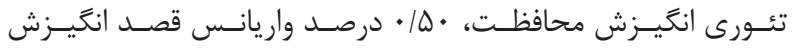

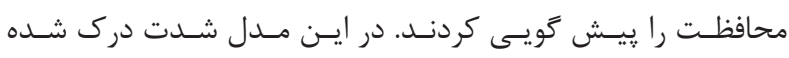

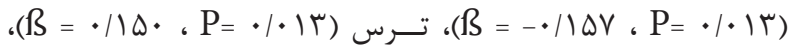

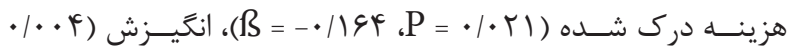

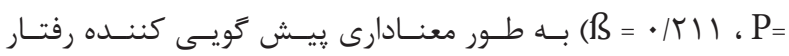

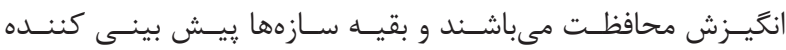

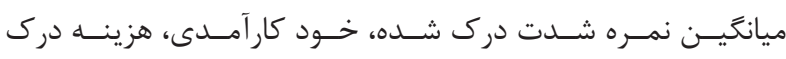

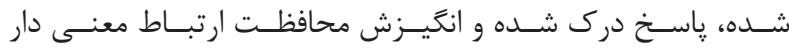

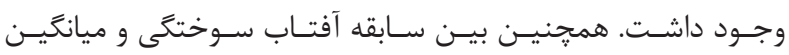

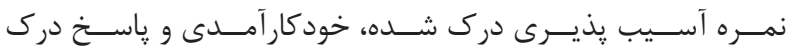

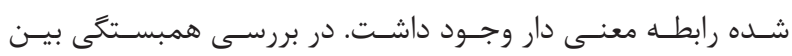

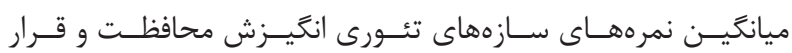

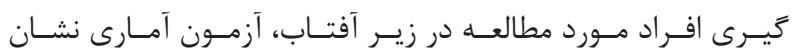

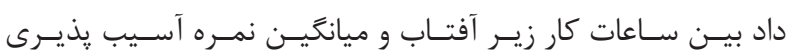

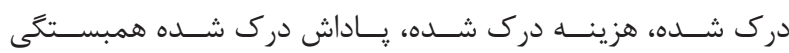

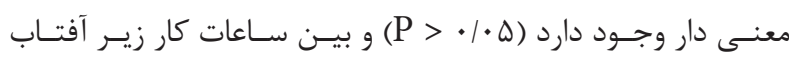

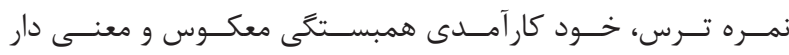

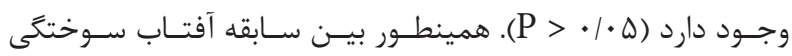

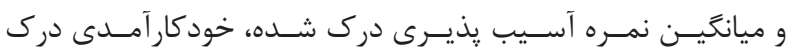

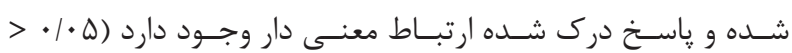
P

\begin{tabular}{|c|c|c|c|c|c|}
\hline \multirow[b]{3}{*}{ 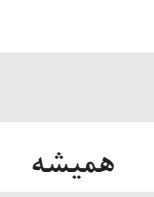 } & \multirow[b]{3}{*}{ بيشتر مواقع } & \multirow{3}{*}{ تعداد (درصد) } & \multicolumn{3}{|c|}{ جدول ": شاخصهاى آمارى رفتارهاى بيشگيرى كننده از سرطان يوست در زنان روستايى } \\
\hline & & & & & 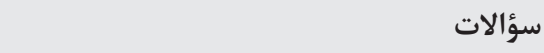 \\
\hline & & & كَاهى اوقات & 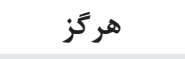 & \\
\hline$I r(\% \vee / \Delta)$ & $\forall \wedge(\% r \cdot / 9)$ & Gr(\%rV) & $V \backslash(\% r \cdot / 9)$ & rद (\%\Q/V) & استفاده از كلاه نقاب دار \\
\hline$\Delta \cdot(\% Y \backslash / V)$ & $V r(\% r I / r)$ & $r \Delta(\% \backslash \Delta / r)$ & $\Delta T(\% T r / 9)$ & r) $(\% 9 / 1)$ & استفاده از كرم ضد افتاب \\
\hline $11(\% / A)$ & $r \Delta(\% \backslash \Delta / r)$ & $\Delta F(\% r r / \Delta)$ & $\Lambda r(\% r \Delta / V)$ & $\uparrow \wedge(\% r \cdot / 9)$ & استفاده از دستكش \\
\hline$\|(\% \leftarrow / \Lambda)$ & $r F(\%) \cdot / F)$ & rG (\%\Q/V) & $\Delta V(\% Y r / \Lambda)$ & $1 \cdot r(\% F F / r)$ & استفاده از عينك آفتابى \\
\hline sY $(\% T V / \Lambda)$ & rq(\%।V) & $\varphi q(\% r \cdot)$ & $99(\% r \cdot)$ & $\mid r(\% \Delta / r)$ & را ميى يوشاند. لباس هايى كه قسمت بيشترى از بدن \\
\hline$r G(\% \backslash \Delta / V)$ & $9 \wedge(\%$ \%/9) & $99(\% r \cdot)$ & $\mathcal{Y V}(\% r \cdot / \varphi)$ & $1 \cdot(\% / T)$ & كار كردن در ساعات اوليه صبح و بعد ظهر \\
\hline$f \cdot(\% \mid V / F)$ & VG(\%rr) & $99(\% ץ \cdot)$ & $r \Delta(\% \backslash \Delta / r)$ & $1 \cdot(\% / T)$ & مراجعه به يزشك در صورت مشاهده علائم \\
\hline ५ (\%।V) & $V r(\% r I / r)$ & $\vee(\% r \cdot / 9)$ & FI $(\% \mathrm{~V} / \mathrm{A})$ & $V(\%)$ & كمتر قرار گرفتن در معرض نور خورشيد \\
\hline
\end{tabular}

\begin{tabular}{|c|c|c|c|c|c|}
\hline \multicolumn{6}{|c|}{ جدول f: مدل ركرسيون خطى پيش كويى كننده رفتار انخيزش محافظت از سرطان يوست در زنان روستايى } \\
\hline $\mathbf{p}$ & $\mathbf{t}$ & B & SE & B & متغير \\
\hline$\cdot 1 \cdot \cdot 1$ & $9 / \wedge \Delta \Delta$ & & $r / 91 \cdot$ & $19 / 94 \wedge$ & مقدار ثابت \\
\hline$\cdot 1 \cdot 11$ & $-r / 4 q 1$ & $-\cdot / 1 \Delta V$ & $\cdot / 191$ & $-\cdot / 4 V V$ & شدت درى شده \\
\hline$\cdot 1 \cdot 11$ & $r / \Delta \cdot \cdot$ & $\cdot / 10$ & $\cdot / 1 \cdot r$ & $\cdot / r \Delta V$ & ترس \\
\hline$\cdot|\cdot r|$ & - T/KTی & $-\cdot 1194$ & $\cdot / \cdot \vee \wedge$ & $-\cdot / 1 \wedge r$ & هزينه درى شده \\
\hline$\cdot 1 \cdot \cdot r$ & $-r / \wedge \wedge q$ & $\cdot|r| 1$ & . & $\cdot / r 91$ & انخَيزش \\
\hline
\end{tabular}


هـر جقــدر شـدت انخيـزش محافظـت درك شـده زنـان روسـتايى

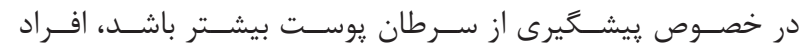

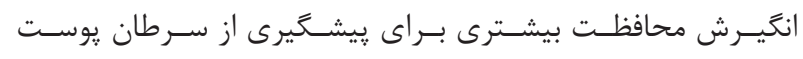

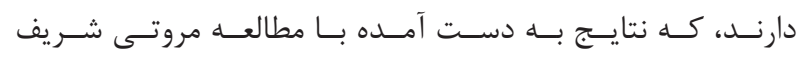

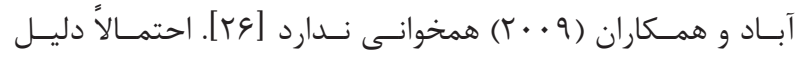

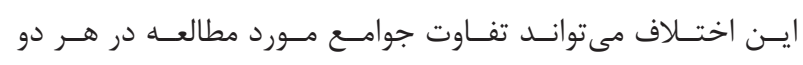

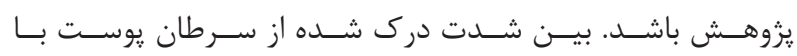

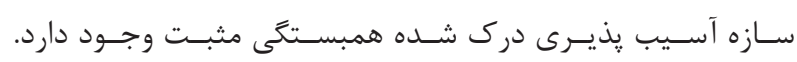

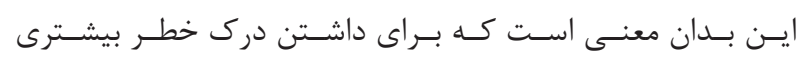

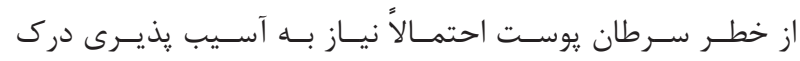

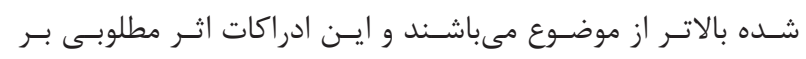

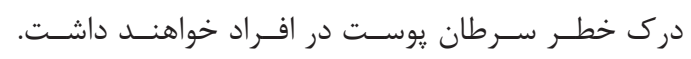

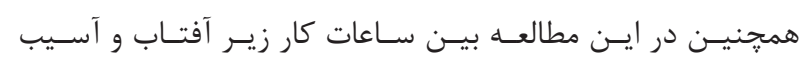

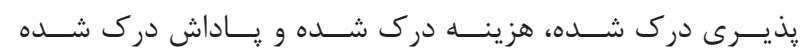

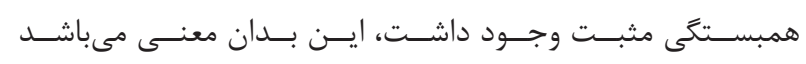

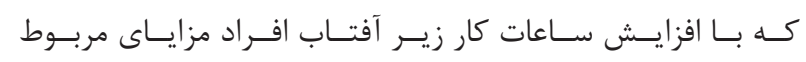

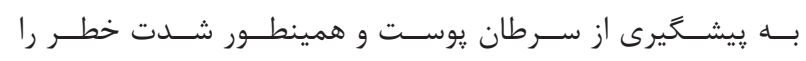

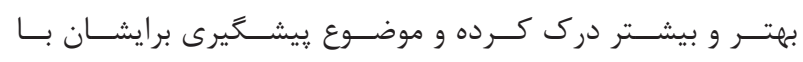

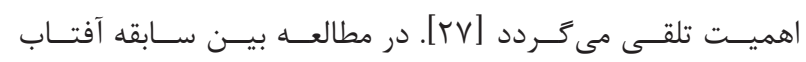

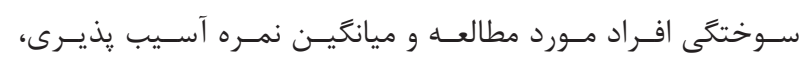

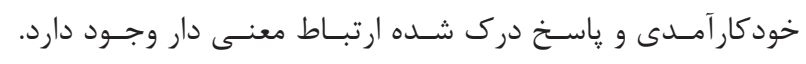

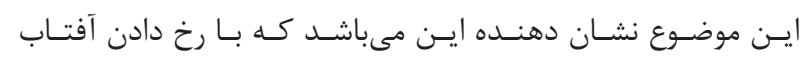

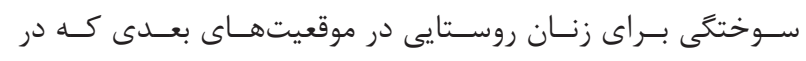

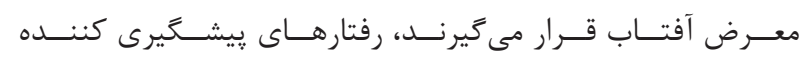

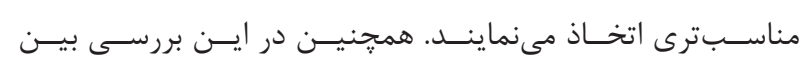

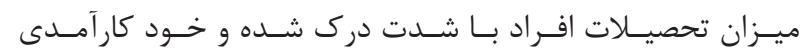

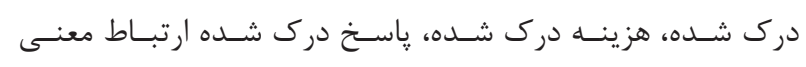

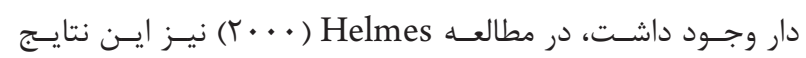

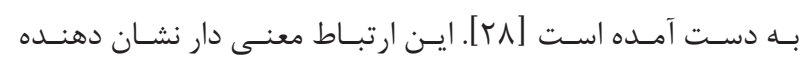

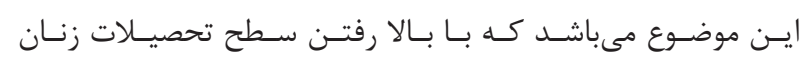

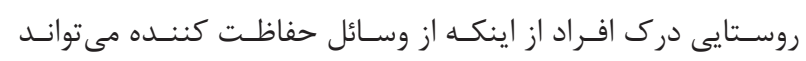

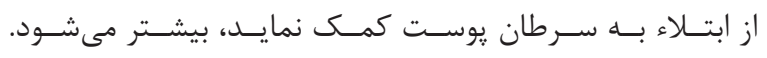

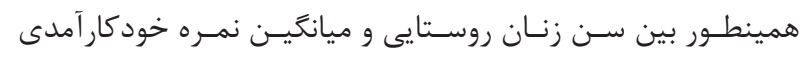

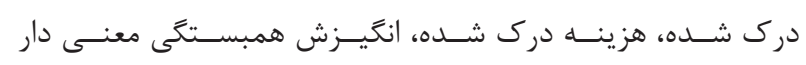

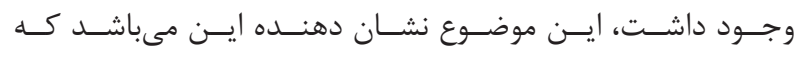

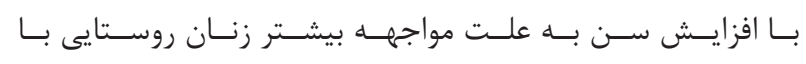

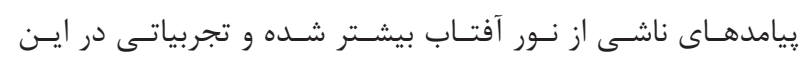

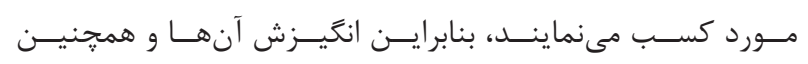

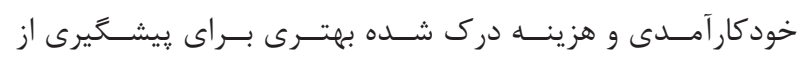

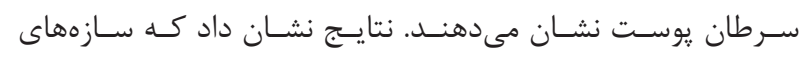

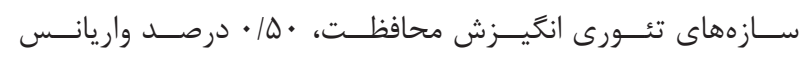

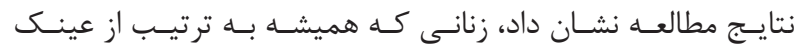

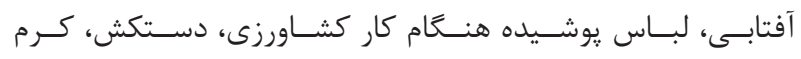

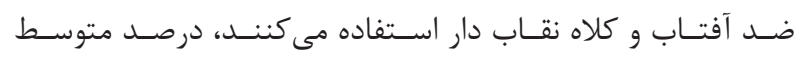

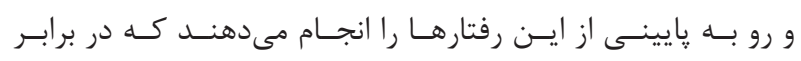

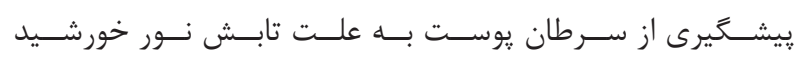

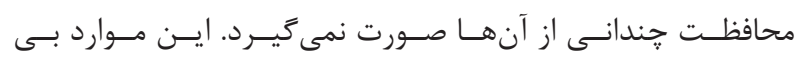

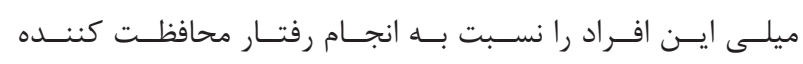

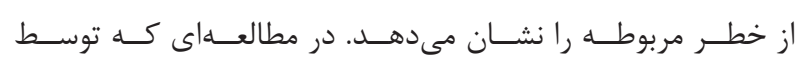

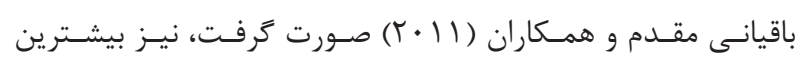

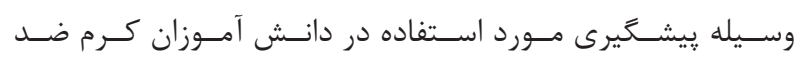

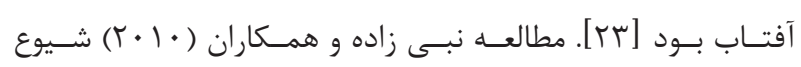

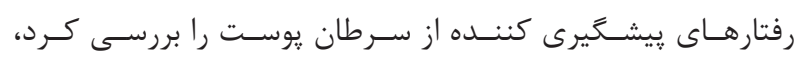

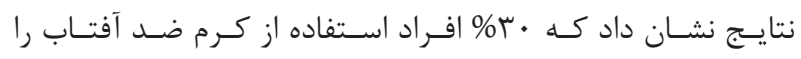

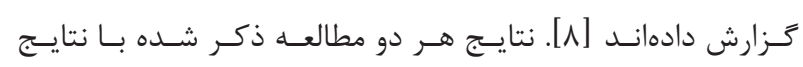

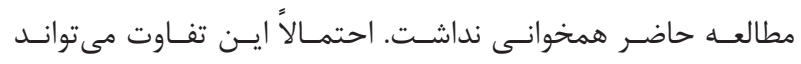

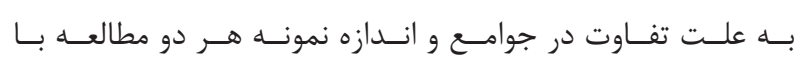
مطالعـهـ حاضـر باشـد.

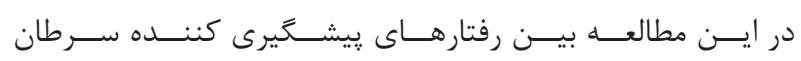

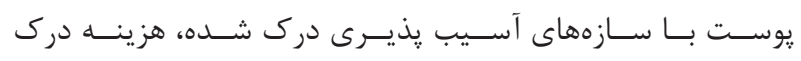

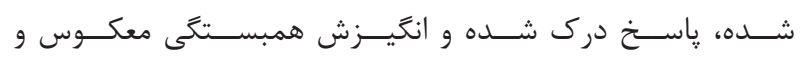

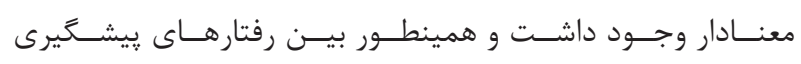

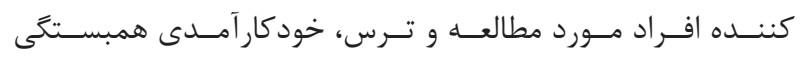

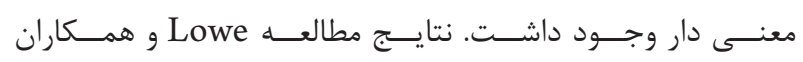

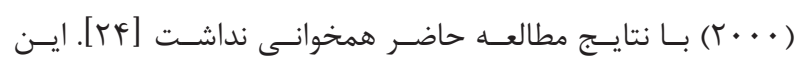

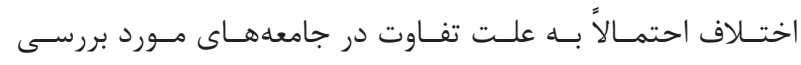

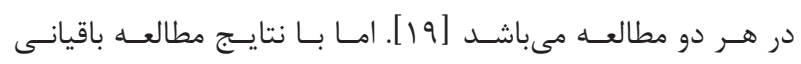

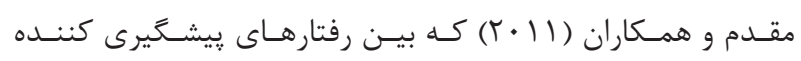

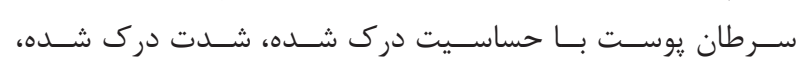

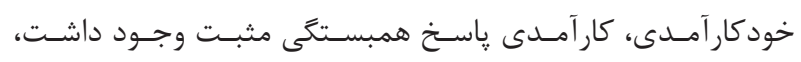

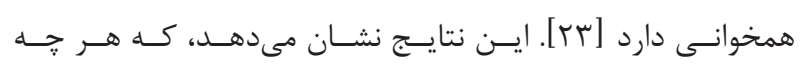

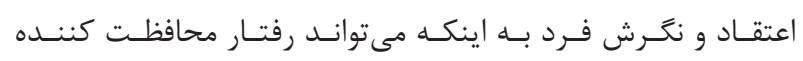

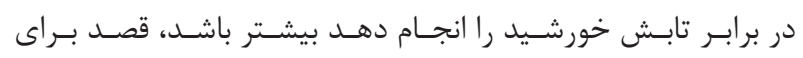

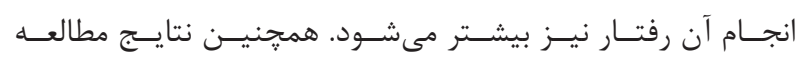

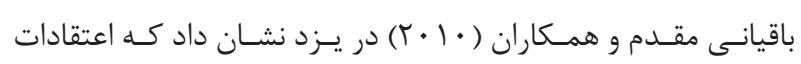

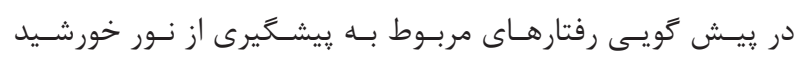

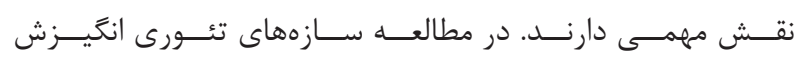

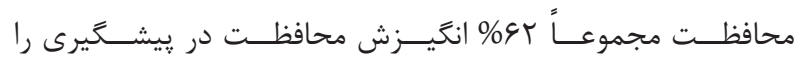

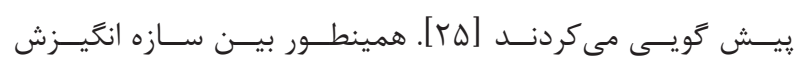

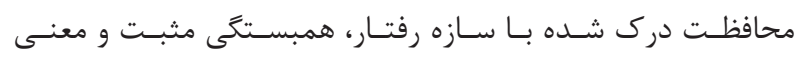

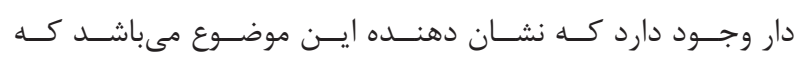




\section{نتيجه كيرى}

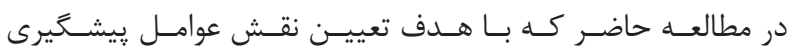

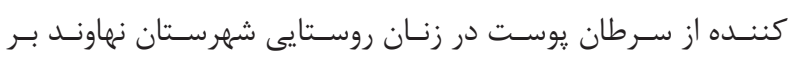

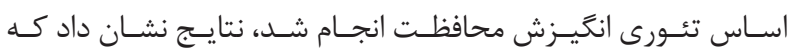

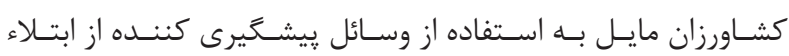

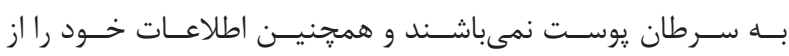

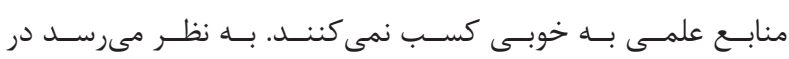

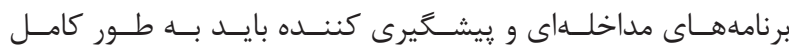

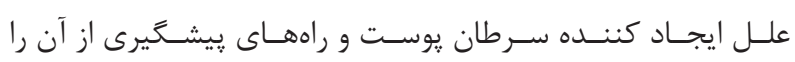

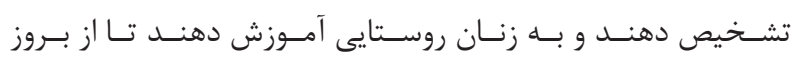

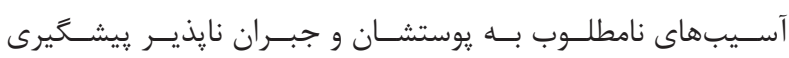

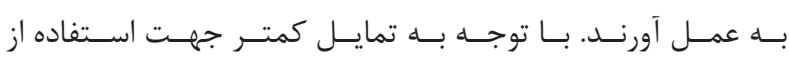

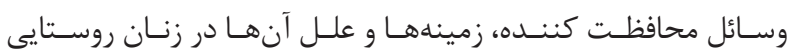

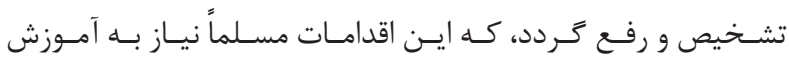

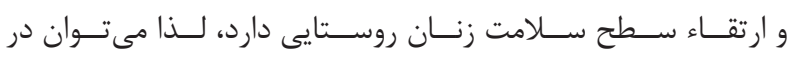

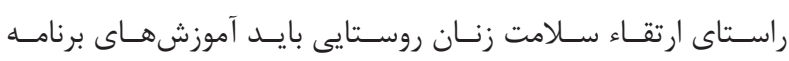

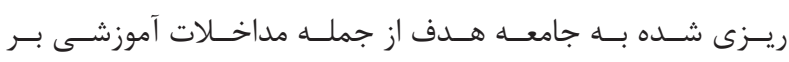

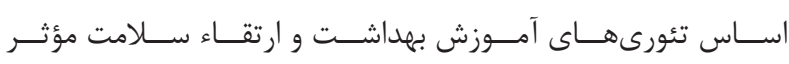

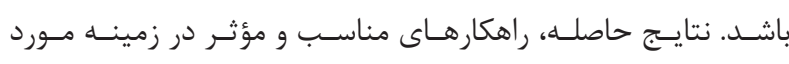

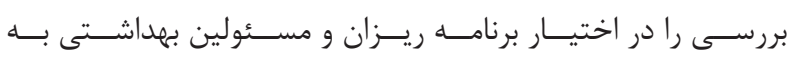

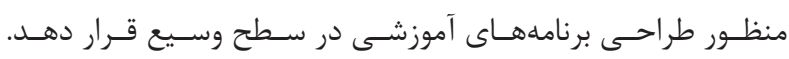

\section{تضاد منافع}

هيج گَونه تضاد منافعى در اين مطالعه وجود ندارد.

\section{سِاسكزارى}

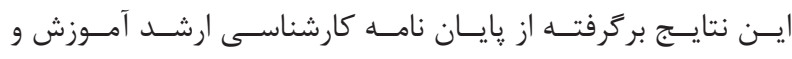

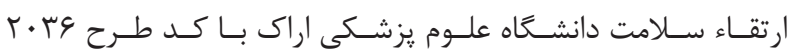

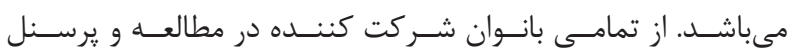

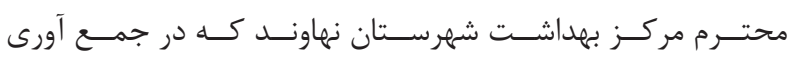

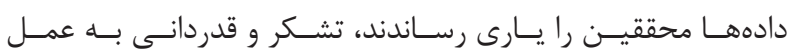

\section{REFERENCES}

مى آيــد.

1. Marjani A, Kabir MJ. Male skin cancer incidence in Golestan province, Iran. J Pak Med Assoc. 2009;59(5):287-9. PMID: 19438130

2. Siegel RL, Miller KD, Jemal A. Cancer statistics, 2016. CA Cancer J Clin. 2016;66(1):7-30. DOI: 10.3322/caac.21332 PMID: 26742998

3. Azizi F, Hatami H, Janghorbani M. [Epidemiology of Common Disorders in Iran]. 2nd ed. Tehran2001.

4. Cokkinides VE, Weinstock MA, O'Connell MC, Thun MJ. Use of indoor tanning sunlamps by US youth, ages 11-18 years, and by their parent or guardian caregivers: prevalence and correlates. Pediatrics. 2002;109(6):1124-30. PMID: 12042553

5. Saraiya M, Hall HI, Uhler RJ. Sunburn prevalence among adults in the United States, 1999. Am J Prev Med. 2002;23(2):91-7. PMID: $\underline{12121796}$

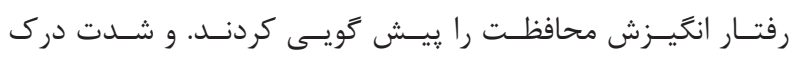

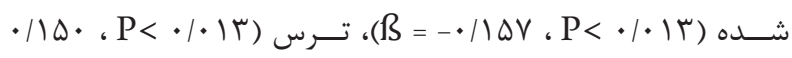

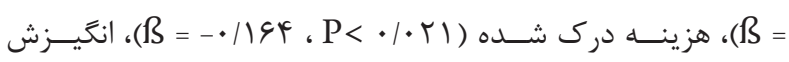

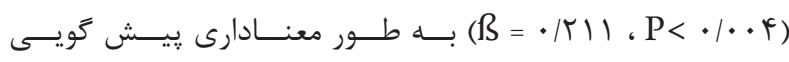

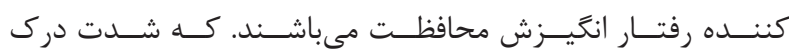

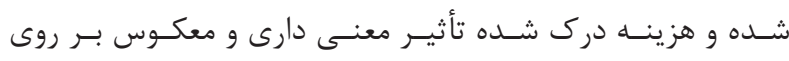

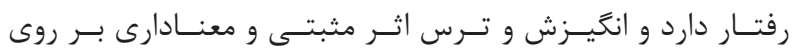
رفتـار دارد. زنـان شـركت كنـــده در مطالعـه در مـورد خطــر سـرطان يوسـت

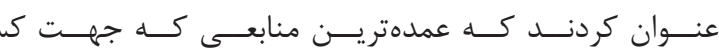

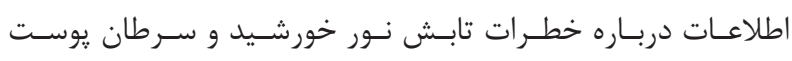

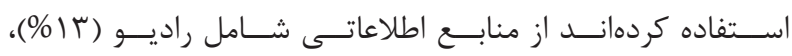

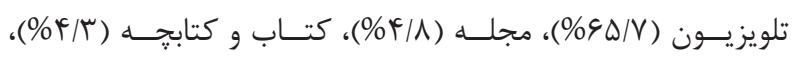

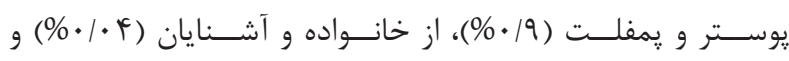

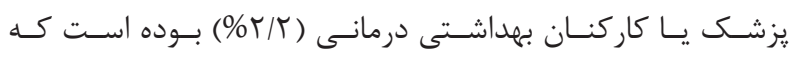

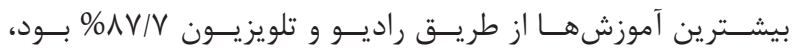

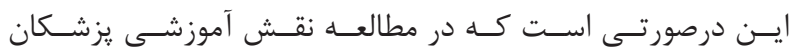

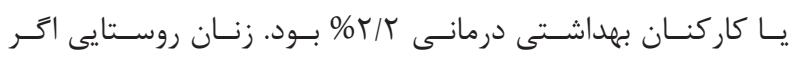

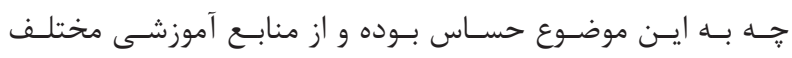

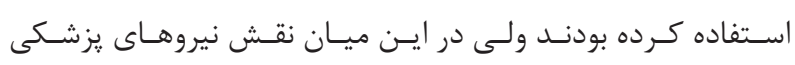

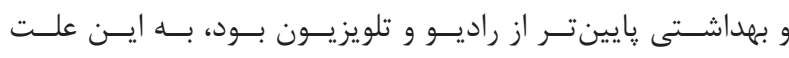

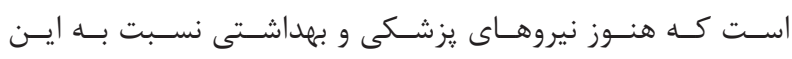

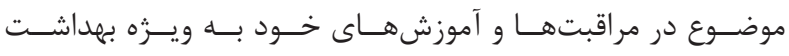

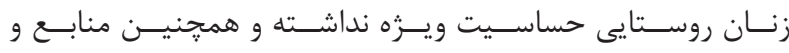

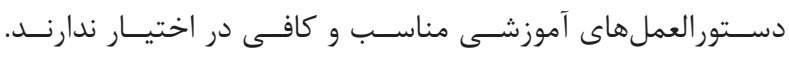

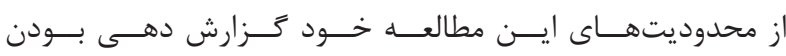

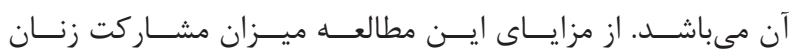

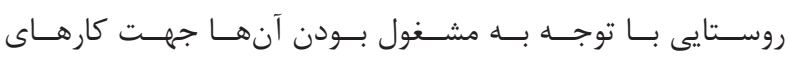

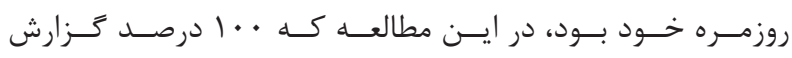

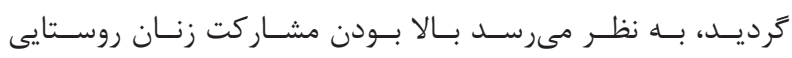

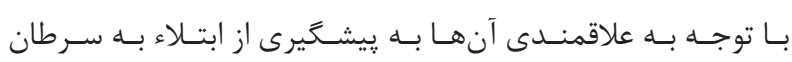

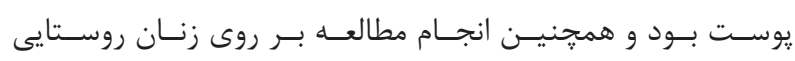

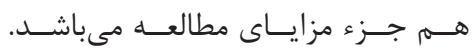

6. Afshari M, Afshari M, Bahrami M, Kangavari M. Factors preventing skin cancer in farmers from tuyserkan city based on protection motivation theory. Iran Occup Health J. 2016;13(1):75-82.

7. Noorbala M. [Evaluation Of skin cancers in Yazd]. Skin Dis. 2007;10(39):13-9.

8. Nabizadeh R, Salehi S, Younesian M, Naddafi K. [Evaluation of the Relationship Between Global Ultraviolet Indea in Different Regions of Iran with Skin Cancer in 1383]. Iranian J Health Environ. 2010;2(4):258-67.

9. Health Do. State registration of cancer cases reported in 2007. Tehran: Center of Special Ghyrvagyrv disease, cancer office, 2010.

10. Gross CP, Smith BD, Wolf E, Andersen M. Racial disparities in cancer therapy: did the gap narrow between 1992 and 2002? Cancer. 
2008;112(4):900-8. DOI: $10.1002 /$ cncr.23228 PMID: 18181101

11. Housman TS, Feldman SR, Williford PM, Fleischer AB, Jr., Goldman $\mathrm{ND}$, Acostamadiedo JM, et al. Skin cancer is among the most costly of all cancers to treat for the Medicare population.J Am Acad Dermatol. 2003;48(3):425-9. DOI: 10.1067/mjd.2003.186 PMID: 12637924

12. Geller AC, Swetter SM, Brooks K, Demierre MF, Yaroch AL. Screening, early detection, and trends for melanoma: current status (20002006) and future directions. J Am Acad Dermatol. 2007;57(4):55572; quiz 73-6. DOI: 10.1016/j.jaad.2007.06.032 PMID: 17870429

13. Barrett T. Sun awareness and skin cancer prevention in the teen population: using a school based approach in Teaching adolescent selfhealth. Arizona, USA: University of Arizona; 2006.

14. Wakeford R. The cancer epidemiology of radiation. Oncogene. 2004;23(38):6404-28. DOI: 10.1038/sj.onc.1207896 PMID: 15322514

15. Allahverdipoor H. [Passing through traditional health education towards theory oriented health education]. Health Prom Educ Mag. 2005;1(3):75-9.

16. El Dib RP, Silva EM, Morais JF, Trevisani VF. Prevalence of high frequency hearing loss consistent with noise exposure among people working with sound systems and general population in Brazil: a cross-sectional study. BMC Public Health. 2008;8:151. DOI: 10.1186/1471-2458-8-151 PMID: 18462490

17. Schulte PA, Stephenson CM, Okun AH, Palassis J, Biddle E. Integrating occupational safety and health information into vocational and technical education and other workforce preparation programs. Am J Public Health. 2005;95(3):404-11. DOI: 10.2105/ AJPH.2004.047241 PMID: 15727967

18. Cismaru M. Using protection motivation theory to increase the persuasiveness of public service communications. Regina, Italy: Saskatchewan Institute of Public Policy, 2006.

19. McMath BF, Prentice-Dunn S. Protection Motivation Theory and Skin Cancer Risk: The Role of Individual Differences in Responses to Persuasive Appeals. J Appl Soc Psychol. 2005;35(3):621-43. DOI: $\underline{10.1111 / j .1559-1816.2005 . t b 02138 . x}$
20. Baghiani-Moghadam MH, Seyedi-Andi SJ, Shokri-Shirvani J, Khafri S, Ghadimi R, Parsian H. Efficiency of two constructs called "fear of disease" and "perceived severity of disease" on the prevention of gastric cancer: Application of protection motivation theory. Caspian J Intern Med. 2015;6(4):201-8. PMID: 26644893

21. Rahaei Z, Ghofranipour F, Morowatisharifabad MA, Mohammadi E. Determinants of Cancer Early Detection Behaviors:Application of Protection Motivation Theory. Health Promot Perspect. 2015;5(2):138-46. DOI: 10.15171/hpp.2015.016 PMID: 26290829

22. Mazloomy Mahmoodabad SS, Noorbala MT, Rahaee Z, Mohammadi M. Knowledge, attitude and performance study of secondary school teachers of Yazd city regarding skin cancer. J Eur Acad Dermatol Venereol. 2010;24(4):424-8. DOI: 10.1111/j.1468-3083.2009.03431.x PMID: 19778358

23. Baghianimoghadam MH, Mohammadi S, Mazloomi Mahmoudabad SS, Norbala MT. [The effect of education based on protection-motivation theory on skin cancer preventive practices among female high school students in Yazd]. Horiz Med Sci. 2011;17(1):27-34.

24. Lowe JB, Borland R, Stanton WR, Baade P, White V, Balanda KP. Sunsafe behaviour among secondary school students in Australia. Health Educ Res. 2000;15(3):271-81. PMID: 10977375

25. Baghianimoghaddam MH, Mohammadi S, Norbala MT, Mazloomi SS. [The study of factors relevant to skin cancer preventive behavior in female high school students in Yazd based on protection motivation theory]. Knowledge Health. 2010;5(1):10-5.

26. Morovati Sharifabad M, Jozi F, Barkhordari A, Falah Zade H. [Factors associated with the use of protective devices for workers in textile factories in Yazd city based on protection motivation theory]. J Iran Occup Health. 2009;6(3):47-54.

27. Raymond DM, 3rd, Lusk SL. Staging workers' use of hearing protection devices: application of the transtheoretical model. AAOHN J. 2006;54(4):165-72. PMID: 16629006

28. Helmes AW. Application of the protection motivation theory to genetic testing for breast cancer risk. Prev Med. 2002;35(5):453-62. PMID: 12431894 


\title{
The Assessment of the Protection Motivation Theory Construct of Skin Cancer Preventive Behaviors in Rural Women
}

\author{
Abdol hossain Kaviani ${ }^{1}$, Nasrin Roozbahani ${ }^{2,}$, Mahboobeh \\ Khorsandi $^{3}$ \\ ${ }^{1}$ MSc Student, Department of Health Education, Faculty of Health, Arak University \\ of Medical Sciences, Arak, Iran \\ ${ }^{2}$ Assistant Professor, Department of Health Education, Faculty of Health, Arak Uni- \\ versity of Medical Sciences, Arak, Iran \\ ${ }^{3}$ Associate Professor, Department of Health Education, Faculty of Health, Arak Uni- \\ versity of Medical Sciences, Arak, Iran \\ * Corresponding author: Nasrin Roozbahani, Assistant Professor, Department of \\ Health Education, Faculty of Medical Sciences, Arak University of Medical Sciences, \\ Arak,Iran.E-mail:Roozbahani@arakmu.ac.ir
}

DOI: $10.21859 / \mathrm{nmj}-24043$

Received: 12.01 .2016

Accepted: 08.08.2016

\section{Keywords:}

Skin Neoplasms

Sunlight

Rural Population

Protection Motivation Theory

How to Cite this Article:

Kaviani A, Roozbahani N, Khorsandi M. The Assessment of the Protection Motivation Theory Construct of Skin Cancer Preventive Behaviors in Rural Women. Sci J Hamadan Nurs Midwifery Fac. 2016;24(4):229-237.DOI: $10.21859 / \mathrm{nmj}-24043$

(C) 2016 Scientific Journal of Hamadan Nursing \& Midwifery Faculty

\begin{abstract}
Introduction: Rural women due to ongoing out door activities are exposed to sunlight and skin cancer. Protection motivation theory effectively prevents behaviors associated with skin cancer. This study aimed to assess protection motivation theory of skin cancer for preventive behaviors in rural women.

Methods: In this cross-sectional study, 230 rural females from four villages of Nahavand city, with a population of over 1000, were selected randomly. Referring to health centers in selected villages, rural women were selected through simple random sampling. Data on the protection motivation theory was based on a questionnaire and interviews with rural females and then linear regression (stepwise) and Pearson were used to analyze the results. Results: Mean age of the participations was $30.55 \pm 7.50$ years. In the regression model, motivation $(B=0.21, P<0.01)$, perceived severity $(B=-0.16, P<0.01)$, perceived costs $(B$ $=-0.16, P<0.01)$ and fear $(B=-0.15, P<0.01)$ were significant predictors of skin cancer preventive behaviors. This model accounted for $25 \%$ of the variance in these behaviors. Conclusions: According to constructs of the theory associated with protection against the sun, educational programs and health promotion using the theory to increase the motivation of rural women to use protective equipment, is emphasized.
\end{abstract}

